\title{
Search for 4th generation quarks
}

\author{
Sadia Khalil*i \\ Kansas State University \\ E-mail: skhalilefnal.gov
}

\begin{abstract}
We present the results of searches for a new heavy 4th generation and vector-like quarks using leptonic, and semileptonic final states comprising of electron(s) and/or muon(s) and jets. The full sample of $5 \mathrm{fb}^{-1}$ of pp collisions recorded with CMS experiment in 2011 at the center-of-mass energy of $7 \mathrm{TeV}$ has been used. In leptonic final states, two same sign dilepton and trilepton channels have been explored. For semileptonic events, the search for heavy quarks is carried out using events with one lepton, and at least four jets with imbalance in transverse momenta, requiring at least one of the jets identified as originating from the fragmentation of a $b$ quark. No significant excess of events over Standard Model expectations is observed, therefore upper mass limits are set at 95\% C.L.
\end{abstract}

36th International Conference on High Energy Physics

4-11 July 2012

Melbourne, Australia

* Speaker.

${ }^{\dagger}$ A footnote may follow. 


\section{Introduction}

The standard model (SM) of particle physics, with its three generations of quarks and leptons, has been a spectacular success in the last few decades, based on numerous experimental discoveries and precision measurements. However, it is widely believed to be an effective theory that should break down at the scale of $\mathscr{O}(1 \mathrm{TeV})$. There are a number of proposed extensions to the standard model and many of these include an additional generation of quarks and leptons. These new particles are heavily constrained by the precision measurements of the electroweak observables and are mainly influenced by the mass differences between the fourth-generation leptons or quarks $[1,2]$.

In this report three of these recent searches are presented which are either a straightforward extension of the SM [3] or could represent a vector-like quarks (VLQ) model [4] where quarks have a bare mass consistent with gauge invariance. The sequential fourth generation predicts a Higgs boson with a mass of $125 \mathrm{GeV}$ to have cross section five times higher than SM prediction. The higher production cross section is related to the couplings that are largely enhanced in $\mathrm{H} \rightarrow \tau \tau$ channel and strongly suppressed in $\mathrm{H} \rightarrow \gamma \gamma$ channel, which is inconsistent with LHC data and the Higgs-like boson observation at $125 \mathrm{GeV}$ [5]. Nevertheless, fourth generation quarks searches are still important. There exist a number of models with heavy quarks decaying with varying branching fractions, where all searches described in this report assume that a heavy quark decays to it's final state with $100 \%$ branching fraction. Therefore, one should be careful in interpreting these results in terms of VLQ and other such models like little Higgs [6] and warped extra dimensions [7] before ruling out the existence of fourth generation heavy quarks at such higher masses.

The theory cross section for strongly produced fourth generation quarks is calculated using the approximate NNLO calculator, HATHOR [8], where cross sections of electroweak produced fourth generation quarks is taken from the MADGRAPH generator.

\section{Search for the pair $\mathrm{t}^{\prime} \overline{\mathrm{t}}^{\prime} \rightarrow \mathrm{WbW} \overline{\mathrm{b}}$}

The search for the strong pair production of a $\mathrm{t}^{\prime}$ quark and its antiparticle, both decaying into a $\mathrm{W}$ boson and $\mathrm{a} b$ or $\overline{\mathrm{b}}$ quark is carried into semileptonic and dileptonic channel. In this report, the more recent search in semileptonic channel using $5 \mathrm{fb}^{-1}$ of Large Hadron Collider (LHC) data at 7 $\mathrm{TeV}$ is described.

In semileptonic channel, one of the $\mathrm{W}$ boson is assumed to decay to leptons (ev or $\mu v$ ) and the other to a quark-antiquark pair. Events are selected with a single charged lepton $(\mathrm{e} / \mu)$ with transverse momenta, $\mathrm{p}_{\mathrm{T}}^{\text {lepton }}>35$ for electrons and $>35$ for muons, an imbalance in missing transverse momentum, $\mathrm{p}_{\mathrm{T}}^{\text {miss }}>20 \mathrm{GeV}$, and at least four jets with at least one them to be a b-jet. Jets are optimized such that $\mathrm{p}_{\mathrm{T}}^{\text {jets }}>100,90,60$ and $50 \mathrm{GeV}$ for first, second, third and greater or equal to fourth leading jet(s). Table. 1 shows the background cross sections, number of observed events and predicted backgrounds.

To separate the signal from SM background, we look at the tails of the two kinematic variables, $\mathrm{S}_{\mathrm{T}}$, define in Eq. 2.1 as the scalar sum of all the decay constituents in the final state and $M_{f i t}$, which is the fitted mass of the top quark, subject to the constraints $m(l v)=\mathrm{m}(\mathrm{q} \overline{\mathrm{q}})=M_{\mathrm{W}}$ and $m(l v \mathrm{~b})=$ $m(\mathrm{q} \overline{\mathrm{q}} \mathrm{b})$.

$$
\mathrm{S}_{\mathrm{T}}=\mathrm{p}_{\mathrm{T}}^{\text {miss }}+\mathrm{p}_{\mathrm{T}}^{\text {lepton }}+\sum \mathrm{p}_{\mathrm{T}}^{\text {jets }}
$$


Table 1: Background cross sections, number of observed and background events for for the e+jets and $\mu+$ jets samples (normalization of background event numbers - see text). The uncertainties quoted are the statistical uncertainties from the MC simulations.

\begin{tabular}{lccc}
\hline \hline & & $\mathrm{e}+$ jets & $\mu+$ jets \\
\hline Integrated luminosity $(\mathrm{L})$ & & $4.98 \mathrm{fb}^{-1}$ & $4.90 \mathrm{pb}^{-1}$ \\
\hline Background process & Cross section & Events & Events \\
\hline $\mathrm{t} \overline{\mathrm{t}}$ & $154 \mathrm{pb}$ & $3950 \pm 490$ & $5460 \pm 670$ \\
$\mathrm{~W}+$ jets & $31 \mathrm{nb}$ & $462 \pm 55$ & $750 \pm 110$ \\
Single t production & $85 \mathrm{pb}$ & $208 \pm 24$ & $336 \pm 45$ \\
Z+jets, WW, WZ, ZZ & $3.1 \mathrm{nb}$ & $49 \pm 8$ & $69 \pm 11$ \\
Multijets & & $78 \pm 9$ & $5 \pm 5$ \\
\hline Total background & & $4750 \pm 560$ & $6620 \pm 800$ \\
\hline Total observed & 4734 & 6448 \\
\hline \hline
\end{tabular}

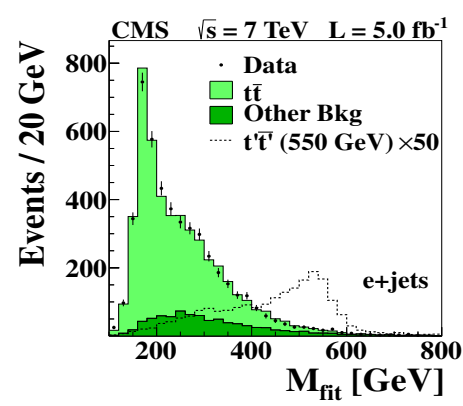

Figure 1: Distribution of $M_{\text {fit }}$ from data, simulation and $\mathrm{t}^{\prime}$ signal with $m_{t^{\prime}}=550 \mathrm{GeV}$ for electron channel.

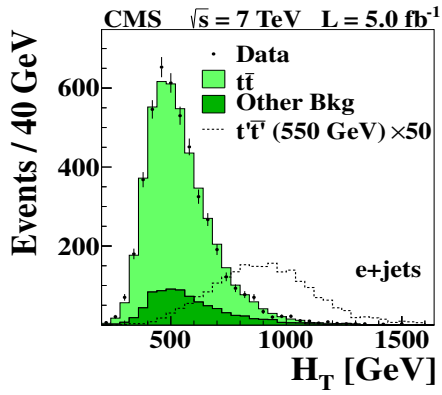

Figure 2: Distribution of $\mathrm{S}_{\mathrm{T}}$ from data, simulation and $\mathrm{t}^{\prime}$ signal with $m_{t^{\prime}}=550 \mathrm{GeV}$ for electron channel.

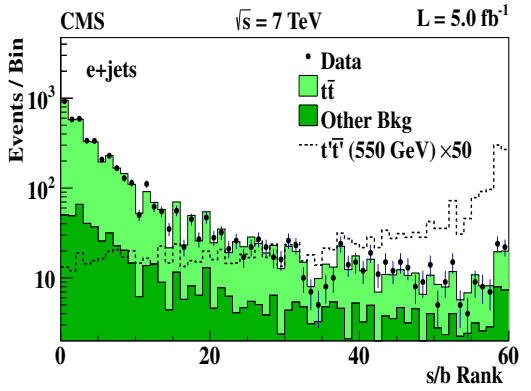

Figure 3: Distributions of expected and observed number of events after the bin merging procedure described in the text for electron channel.

The kinematic reconstruction allows correct jet-quark assignment that maximizes the separation of $\mathrm{t}^{\prime} \bar{t}^{\prime}$ and the $\mathrm{t} \overline{\mathrm{t}}$ background. Figures 1 and 2 shows the projections of the two-dimensional $\mathrm{S}_{\mathrm{T}}$ versus $M_{f i t}$ distribution for data and simulations, that shows a clear separation between signal and background for electron channel. Similar distributions are obtained for muon channel.

However, bins with insufficient bin statistics can lead to incorrect statistical inferences. Hence, bins in two-dimensional histograms of $\mathrm{S}_{\mathrm{T}}$ versus $M_{\text {fit }}$ are combined until the statistical precision in each bin of the background and signal histograms is adequate. Specifically all bins are sorted by their expected signal-to-background ratio, determined from analytical fit to $\mathrm{S}_{\mathrm{T}}$ and $M_{f i t}$ distributions and then merged with similar signal-to-background ratios so that the fractional statistical uncertainty of the signal and the background predictions is below $20 \%$ in all bins. Figure 3 shows the resulting distributions with the ordered in increasing signal-to-background ratio for electron channel, where signal events are clustered towards the right and background events to the left of the distributions. Similar distribution is obtained for muon channel. The search for $\mathrm{t}^{\prime} \mathrm{t}^{\prime}$ is carried using these one-dimensional histograms. 
The computation of the $t^{\prime} t^{\prime}$ cross section limit uses the CLs criterion $[9,10]$ with the asymptotic approximation [11] of the likelihood ratio. The limit calculation includes the following systematic uncertainties. The integrated luminosity has a precision of $2.2 \%$. The $t \bar{t}$ cross section is taken from the CMS measurement of $154 \pm 18 \mathrm{pb}$ [12] and hence constrain to an uncertainty of $11 \%$. Other background are normalized to their predicted yields, with a 50\% uncertainty. The lepton trigger and selection efficiencies have an uncertainty of 3\%. The effect on the normalization of events is accounted due to uncertainty on jet energy calibration and matrix element to parton-matching scale. The largest effects on the expected cross section limits come from the normalization of the electroweak background and the jet energy calibration. Other uncertainties originating from the jet energy resolution, the b-tagging efficiency, the number of additional interactions in the same beam crossing, the factorization scale, and initial and final-state radiation have a negligible effect on the limit calculation.

Figure 4 shows the observed and expected 95\% confidence level (C.L) upper limits for the $\mathrm{t}^{\prime} \mathrm{t}^{\prime}$ cross section as a function of the $\mathrm{t}^{\prime}$-quark mass for the combined e+jets and $\mu+$ jets channels. Therefore, we exclude the existence of chiral fourth generation quark below $570 \mathrm{GeV}$ at $95 \%$ C.L.

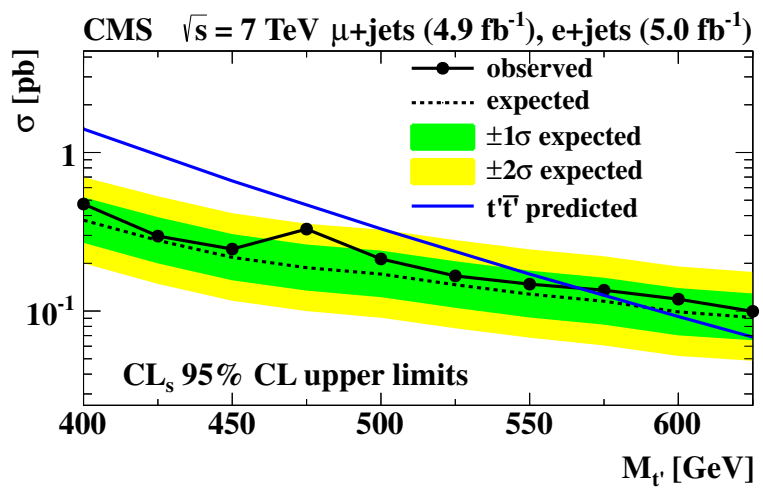

Figure 4: Observed and expected limits for combined e+jets and $\mu+$ jets channels .

\section{Combined search for $\mathrm{t}^{\prime} / \mathrm{b}^{\prime}$-quark production}

This analysis is a combined search for fourth-generation quarks that are produced through the strong interaction, namely $\mathrm{b}^{\prime} \overline{\mathrm{b}^{\prime}}$ and $\mathrm{t}^{\prime} \mathrm{t}^{\prime}$, or through electroweak production, such as $\mathrm{tb}^{\prime}, \mathrm{t}^{\prime} \overline{\mathrm{b}}$ and $\mathrm{t}^{\prime} \overline{\mathrm{b}^{\prime}}$ and their charge conjugates.

This is a model-dependent search with an extended $4 \times 4 C K M$ matrix, $V_{C K M}^{4 \times 4}$ in which up-type an down-type fourth-generation quarks, $\mathrm{t}^{\prime}$ and $\mathrm{b}^{\prime}$ have degenerate masses and hence only one free parameter, $A$, where $0 \leq A \leq 1$ :

$$
V_{C K M}^{4 \times 4}=\left(\begin{array}{cccc}
V_{u d} & V_{u s} & V_{u b} & V_{u b^{\prime}} \\
V_{c d} & V_{c s} & V_{c b} & V_{c b^{\prime}} \\
V_{t d} & V_{t s} & V_{t b} & V_{t b^{\prime}} \\
V_{t^{\prime} d} & V_{t^{\prime} s} & V_{t^{\prime} b} & V_{t^{\prime} b^{\prime}}
\end{array}\right)=\left(\begin{array}{cccc}
\mathscr{O}(1) & \mathscr{O}(0) & \mathscr{O}(0) & \mathscr{O}(0) \\
\mathscr{O}(0) & \mathscr{O}(1) & \mathscr{O}(0) & \mathscr{O}(0) \\
\mathscr{O}(0) & \mathscr{O}(0) & \sqrt{A} & \sqrt{1-A} \\
\mathscr{O}(0) & \mathscr{O}(0) & -\sqrt{1-A} & \sqrt{A}
\end{array}\right)
$$

Within this model mixing is only allowed between the third and the fourth generation and the heavy fourth-generation quarks are assumed to decay in $100 \%$ to the third-generation quarks. The 
cross section of the strongly produced processes, $\mathrm{t}^{\prime} \bar{t}^{\prime}$ and $\mathrm{b}^{\prime} \overline{\mathrm{b}}^{\prime}$ are not related to the value of A. On the other hand, the cross sections of the $\mathrm{t}^{\prime} \overline{\mathrm{b}}$ and $\mathrm{t}^{\prime}$ processes depend linearly on $(1-A)$ and $\mathrm{t}^{\prime} \overline{\mathrm{b}^{\prime}}$ cross section on $A$. Following final states are considered in this analysis, where charges are omitted for simplicity.

- $\mathrm{t}^{\prime} \mathrm{b} \rightarrow \mathrm{bWb}$

- $\mathrm{t}^{\prime} \mathrm{t}^{\prime} \rightarrow \mathrm{bWbW}$

- $\mathrm{b}^{\prime} \mathrm{t} \rightarrow \mathrm{tWbW} \rightarrow \mathrm{bWWbW}$

- $\mathrm{t}^{\prime} \mathrm{b}^{\prime} \rightarrow \mathrm{tWbW} \rightarrow \mathrm{bWWbW}$

- $\mathrm{b}^{\prime} \mathrm{b}^{\prime} \rightarrow \mathrm{tWtW} \rightarrow \mathrm{bWWbWW}$

Events are selected with a single charged lepton $(\mathrm{e} / \mu)$ with $\mathrm{p}_{\mathrm{T}}^{\text {lepton }}>40, \mathrm{p}_{\mathrm{T}}^{\text {miss }}>40 \mathrm{GeV}$, and at least one jet with $\mathrm{p}_{\mathrm{T}}^{\text {jets }}>30$, and requiring at least one of them to be a b-jet. Since each of final decay states consists of at least two b-jets and one to four $\mathrm{W}$ bosons, events are classified based on the number of b-jets (exactly one or at least two) and on the number of W bosons ( one, two, three or at least four) with at least one of them decaying leptonically. The combination of one b-jet and one $\mathrm{W}$ boson is not considered. Hadronically decaying $\mathrm{W}$ bosons, $\mathrm{W} \rightarrow \mathrm{q} \overline{\mathrm{q}}$, are reconstructed by considering all possible jet combinations after removal of $\mathrm{b}$-jets and fitting the reconstructed mass distribution with a Gaussian fit with $M_{f i t}=84.3 \mathrm{GeV}$ and width, $\sigma_{M_{\mathrm{W}}}^{f i t}=9.6 \mathrm{GeV}$. The W boson candidate is chosen whose mass is within a $\pm 1 \sigma_{M_{\mathrm{W}}}^{\text {fit }}$ window around $M_{f i t}$. The jet pair that provides the hadronically decaying $\mathrm{W}$ boson is removed from the jet collection and the procedure is repeated until no more hadronically decaying $\mathrm{W}$ boson candidates are found.

The search is performed in single lepton $(\mathrm{e} / \mu)$ and same-sign dilepton and trilepton decay channels. For ee $/ \mu \mu$ channels, events are rejected if found within $10 \mathrm{GeV}$ of $\mathrm{Z}$ boson mass. At least four (two) jets are required for dilepton (trilepton) channel. Backgrounds such as charge misidentification, fake leptons (single top and semileptonic $t \bar{t}$ decays) are obtained from data and irreducible backgrounds such as $\mathrm{WW}, \mathrm{WZ}, \mathrm{ZZ}, \mathrm{t} \overline{\mathrm{t}}+\mathrm{W}$ and $\mathrm{t}+\mathrm{t}+\mathrm{Z}$ are estimated using simulations. The total number of expected background events for the same-charge dilepton and trilepton events is given in Table 2 .

Table 2: The prediction for the total number of background events is compatible with the number of observed events in the same-sign dilepton and the trilepton subsamples.

\begin{tabular}{lcccc}
\hline \hline type & 2 muons & 2 electrons & electron+muons & trilepton \\
\hline Observed & 2 & 2 & 2 & 1 \\
Background & $0.83 \pm 0.11$ & $1.36 \pm 0.19$ & $2.27 \pm 0.22$ & $0.96 \pm 0.12$ \\
Signal $\left(\mathrm{A}=1, m_{q^{\prime}}=550 \mathrm{GeV}\right)$ & $3.31 \pm 0.15$ & $2.03 \pm 0.36$ & $5.29 \pm 0.19$ & $3.37 \pm 0.16$ \\
Signal $\left(\mathrm{A}=0.8, m_{q^{\prime}}=550 \mathrm{GeV}\right)$ & $3.79 \pm 0.15$ & $2.29 \pm 0.36$ & $6.00 \pm 0.19$ & $3.65 \pm 0.16$ \\
\hline \hline
\end{tabular}

In the single lepton subsamples with one or three $\mathrm{W}$ bosons, we use $\mathrm{S}_{\mathrm{T}}$ to separate signal from $\mathrm{SM}$ background, where $\mathrm{S}_{\mathrm{T}}$ is defined as the scalar sum of the transverse momenta of the reconstructed objects in the final state. 


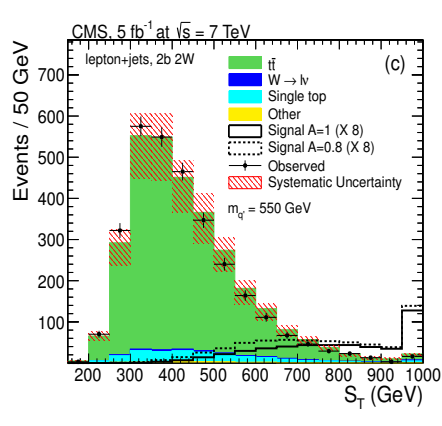

Figure 5: Distribution of $S_{T}$ for two b-jets and two $\mathrm{W}$ bosons compared to their expectation from the simulation assuming the fitted nuisance parameters.

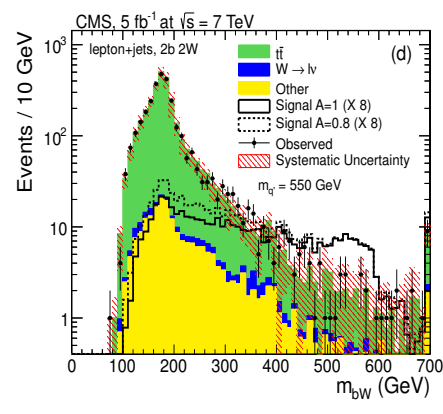

Figure 6: Distribution of $M_{\mathrm{bW}}$ for two b-jets and two $\mathrm{W}$ bosons compared to their expectation from the simulation assuming the fitted nuisance parameters.

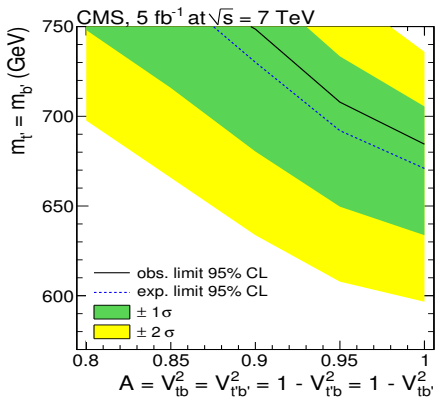

Figure 7: Exclusion limit on $m_{\mathrm{t}^{\prime}}$ and $m_{\mathrm{b}^{\prime}}$ as a function of the $V_{C K M}^{4 \times 4}$ parameter A. Values below the line are excluded at $95 \%$ confidence level (CL) as described in text.

$$
\mathrm{S}_{\mathrm{T}}=\mathrm{p}_{\mathrm{T}}^{\mathrm{miss}}+\mathrm{p}_{\mathrm{T}}^{\text {lepton }}+\mathrm{p}_{\mathrm{T}}^{\mathrm{jet}}+\mathrm{p}_{\mathrm{T}}^{\mathrm{b}-\mathrm{jet}}+\sum_{\mathrm{i}=0}^{\mathrm{N}} \mathrm{p}_{\mathrm{T}}^{\mathrm{W}_{\mathrm{q}}^{\mathrm{i}} \overline{\bar{\mu}}}
$$

where the sum runs over the number of reconstructed hadronically decaying $\mathrm{W}$ bosons. In case there is no second $b$-jet in the event, $\mathrm{p}_{\mathrm{T}}^{\text {jet }}$ is the leading jet $\mathrm{p}_{\mathrm{T}}$ which is not used in hadronic $\mathrm{W}$ reconstruction. For subsamples with two $\mathrm{W}$ bosons, the mass of the hadronic bW system, $M_{\mathrm{bW}}$ is also considered in addition to $S_{T}$. Four jets need to be assigned to the quarks to reconstruct the final state $\mathrm{t}^{\prime} \mathrm{t}^{\prime} \rightarrow \mathrm{WbWb} \rightarrow \mathrm{q} \overline{\mathrm{q}} \mathrm{b} l v l \mathrm{~b}$ and hence $M_{\mathrm{bW}}$. Therefore, six observables such as angles between the decay products, the $\mathrm{W}$ boson mass, the $\mathrm{p}_{\mathrm{T}}$ of the hadronically decaying top quark and a variable related to b-jet identification are used for a correct jet-quark assignment with the largest value of the likelihood ratio. Figures 5 and 6 shows the projections of the two-dimensional $\mathrm{S}_{\mathrm{T}}$ versus $M_{\mathrm{bW}}$ distributions, which along with other categories are used to infer the existence of signal. The sources of systematics considered are similar as described for $t^{\prime} \bar{t}^{\prime} \rightarrow W b W \bar{b}$ search in section 2, except the additional systematics due to same-sign dilepton background estimation.

The $C L_{s}$ procedure $[9,10,11]$ is used to calculate the combined limit for the single muon, single electron, same-sign dilepton and trilepton channels. The point $\left(A, m_{\mathrm{q}^{\prime}}\right)$ at the $95 \% \mathrm{CL}$ is excluded if the upper limit on the combined cross section variable is smaller than its predicted value within the fourth generation model. The procedure is repeated for each value of $A$ and $m_{\mathrm{q}^{\prime}}$. Therefore, fourth-generation quarks with a degenerate mass below $685 \mathrm{GeV}$ are excluded at the 95\% CL for a parameter value of $A \sim 1$ as shown in Figure 7. The slope indicates the sensitivity of the analysis to the $\mathrm{t}^{\prime} \mathrm{b}$ and $\mathrm{tb}^{\prime}$ processes. For a mass difference of $m_{\mathrm{t}^{\prime}}-m_{\mathrm{b}^{\prime}}=25 \mathrm{GeV}$, the exclusion limit shifts by $20 \mathrm{GeV}$.

\section{Search for $B \rightarrow b Z$ in dilepton channel}

This is a search for a heavy vector-like B quark, which would manifest itself as a resonance in the $M_{\mathrm{bZ}}$ distribution, where $M_{\mathrm{bZ}}$ represents the invariant mass of a state consisting of a b-jet and a $\mathrm{Z}$ boson. The $\mathrm{Z}$ boson candidates decaying through $\mathrm{Z} \rightarrow \mathrm{e}^{+} \mathrm{e}^{-}$and $\mathrm{Z} \rightarrow \mu^{+} \mu^{-}$modes are used 
in the detection of the $\mathrm{B}$ quark. It is assumed that the $\mathrm{B}$ and $\overline{\mathrm{B}}$ quarks are produced in pairs with $\mathrm{BR}(\mathrm{B} \rightarrow \mathrm{bZ})=100 \%$.

Event selection requires two electrons with $\mathrm{p}_{\mathrm{T}}>25 \mathrm{GeV}$ and two muons with $\mathrm{p}_{\mathrm{T}}>20 \mathrm{GeV}$. $\mathrm{Z}$ boson candidates are defined by a pair of opposite sign charged leptons and an invariant mass 60 $\mathrm{GeV}<M_{l^{+} l^{-}}<120 \mathrm{GeV}$. Events are also required to have at least 2 jets with $\mathrm{p}_{\mathrm{T}}^{\mathrm{jets}}>30 \mathrm{GeV}$, and one of them to be a b-jet. Fake jets due to mis-identified leptons were largely removed by requiring that the jets are well-separated from reconstructed electrons or muons. B quark candidate events are further required to have at least one $\mathrm{Z}$ boson with $\mathrm{p}_{\mathrm{T}}>95 \mathrm{GeV}$ and at least one b-jet with $\mathrm{p}_{\mathrm{T}}>65$ $\mathrm{GeV}$. Corrections to $\mathrm{MC}$ are applied due to Jet energy scales, trigger and lepton identification and isolation. A residual correction factor ( 0.94 for electrons and 1.01 for muons) is obtained by floating the normalization of the background yield to a fit in data and is also applied. Events yields for signal and background processes, after the full event selection, are shown in Table 3.

Table 3: SM background process theory cross section and data after the full event selection for the $\mathrm{Z} \rightarrow \mathrm{e}^{+} \mathrm{e}^{-}$ and $\mathrm{Z} \rightarrow \mu^{+} \mu^{-}$channels.

\begin{tabular}{lccc}
\hline \hline Process & Cross section $(\mathrm{pb})$ & $\mathrm{Z} \rightarrow \mathrm{e}^{+} \mathrm{e}^{-}$ & $\mathrm{Z} \rightarrow \mu^{+} \mu^{-}$ \\
\hline $\mathrm{Z}+\mathrm{jets}$ & 2939 & $557 \pm 15$ & $847 \pm 25$ \\
$\mathrm{t}+\mathrm{jets}$ & 168 & $80 \pm 4$ & $137 \pm 7$ \\
$\mathrm{~W}^{+} \mathrm{W}^{-}$ & 43 & $0.10 \pm 0.10$ & $0.17 \pm 0.11$ \\
$\mathrm{~W}^{ \pm} \mathrm{Z}$ & 18 & $3.6 \pm 0.3$ & $6.1 \pm 0.5$ \\
$\mathrm{ZZ}$ & 5.9 & $5.9 \pm 0.2$ & $9.2 \pm 0.3$ \\
\hline Total & & $648 \pm 15$ & $999 \pm 26$ \\
\hline Data & & $604 \pm 24$ & $928 \pm 30$ \\
\hline \hline
\end{tabular}

The mass spectrum of the selected bZ candidates is used to search for a resonance peak consistent with that from a B quark. The distribution of the invariant mass of the bZ candidates is shown in Figures. 8 and 9 for the muon and electron channels. The points with error bars are data; the open histograms are the expected signals for a B quark of mass $350 \mathrm{GeV}$; the filled histograms are the expected contributions from the $\mathrm{Z}+\mathrm{jets}, \mathrm{t}+\mathrm{j}$ ets, and diboson background processes. The histogram corresponding to the B quark signal is stacked on top of the background contributions. The shaded areas represent the quadratic sum of the statistical and systematic uncertainties of the total background.

Systematics due to backgrounds and above corrections are considered in a similar way as in other two analysis described in sections 3 and 2. The largest one comes from Z+jets, where a light quark or gluon jet fakes a b-jet. Therefore, separate cross-section uncertainties are assigned to $\mathrm{Z}+\mathrm{b}, \mathrm{Z}+\mathrm{c}$, and $\mathrm{Z}+$ light $(\mathrm{u}, \mathrm{d}, \mathrm{s}, \mathrm{g})$ flavour jets. The uncertainty in the $\mathrm{Z}+\mathrm{b}$ inclusive cross section is $+13 \% /-16 \%$ and same are considered for $Z+c$ cross section. The uncertainty for $Z+l i g h t$ flavour jets is taken as $4 \%$. For $t \bar{t}+j$ ets background events, the cross section uncertainty is assumed to be $14 \%$. Additional systematic due to shape of $\mathrm{Z}+$ jets backgrounds is determined by comparing the two distributions using different event generators, SHERPA and MADGRAPH.

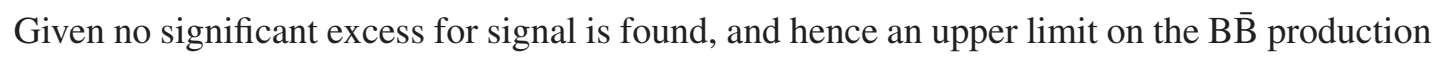




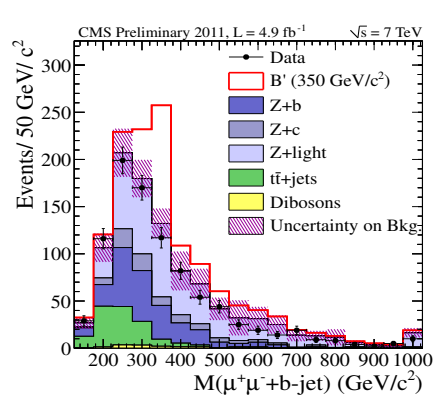

Figure 8: The invariant mass of B quark candidates for the muon channel as described in text.

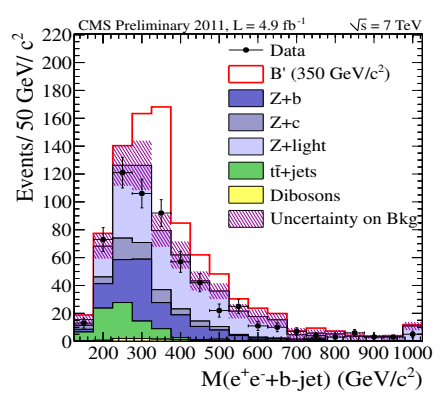

Figure 9: The invariant mass of B quark candidates for the electrons as described in text.

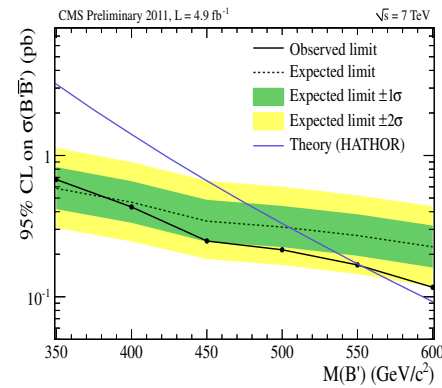

Figure 10: The $95 \%$ exclusion limit on B quark mass calculated using the combine $\mathrm{Z} \rightarrow \mu^{+} \mu^{-}$ and $\mathrm{Z} \rightarrow \mathrm{e}^{+} \mathrm{e}^{-}$channels.

cross section is calculated for various masses of the B quark, by assuming $\mathrm{BR}(\mathrm{B} \rightarrow \mathrm{bZ})=100 \%$. The modified-frequentist (CLs) approach $[9,10,11]$ is used to place a 95\% C.L upper limit on the cross section. Figure 10 shows that a B quark with mass below $510 \mathrm{GeV}$ is excluded.

\section{Summary}

CMS has the most stringent limits on the existence of the fourth generation quarks and we have reached the critical mass of $\sim 550 \mathrm{GeV}$, at which fermions weak interactions becomes nonperturbative [13]. More details about the above presented searches can be found in literature [14, $15,16]$. A summary of all fourth generation quarks searches at CMS can be found in CMS B2G group, physics public results page [17].

\section{References}

[1] H. Flaecher, et al., Revisiting the Global Electroweak Fit of the Standard Model and Beyond with Gfitter, Eur. Phys. J C60 (543) 009 [arXiv: 0811.0009 ].

[2] M. Buchkremer, et al., Closing in on a perturbative fourth generation, [arXiv: 1204.5403 ].

[3] P. H. Frampton. et al., Quarks and Leptons Beyond the Third Generation, Phys. Rep 330 (263) 00, [hep-ph/9903387].

[4] B. A. Dobrescu. et al., Prospects for top-prime quark discovery at the Tevatron, JHEP 906 (001) 09, [arXiv:0902.0792].

[5] CMS Collaboration., Observation of a new boson at a mass of $125 \mathrm{GeV}$ with the CMS experiment at the LHC, Phys. Lett. B $\mathbf{7 1 6}$ (30) 12 [arXiv:1207. 7235].

[6] M. Schmaltz. et al., Introducing the Little Higgs, Nucl. Phys. Proc. Suppl 117 (40) 03, [hep-ph/0210415].

[7] A Large mass hierarchy from a small extra dimension, Phys. Rev. Lett 83 (3370) 99, [hep-ph/9905221].

[8] M. Aliev et al., - HATHOR - HAdronic Top and Heavy quarks crOss section calculatoR, Comput. Phys. Commun. 182 (1034) 11, [arXiv: 1007.1327$].$ 
[9] T. Junk, Confidence level computation for combining searches with small statistics, Nucl. Instrum. Meth $\mathbf{A 4 3 4}$ (435) 99, [hep-ex/9902006].

[10] A. L. Read, Presentation of search results: the CLs technique, J. Phys. G28 (2693) 02.

[11] G. Cowan; Asymptotic formulae for likelihood-based tests of new physics, et al., Eur. Phys. J C71 (1554) 11, [arXiv:1007.1727].

[12] S. Chatrchyan et al. (CMS), Measurement of the t $\bar{t}$ Production Cross Section in pp Collisions at $7 \mathrm{TeV}$ in Lepton + Jets Events Using b-quark Jet Identification, Phys. Rev. D 84 (092004) 11, [arXiv:1108.3773v1].

[13] M.S. Chanowitz, et al., Phys. Lett. B 78, (285) 78.

[14] CMS Collaboration, Search for pair produced fourth-generation up-type quarks in pp collisions at $\operatorname{sqrt}(s)=7 \mathrm{TeV}$ with a lepton in the final state, Phys. Lett. B 718 (307) 12, [arXiv: 1209.0471 ] .

[15] CMS Collaboration, Combined search for the quarks of a sequential fourth generation, Submitted to Phys. Rev. D, [arXiv:1209.1062].

[16] CMS Collaboration, Search for a Vector-like Quark with Charge 2/3 in $t+Z$ Events from $p p$ Collisions at sqrt(s) = 7 TeV. Phys Rev Lett 107 (271802) 11, [arXiv:1109.4985].

[17] B2G physics group public results, CMS Collaboration, https://twiki.cern.ch/twiki/bin/view/CMSPublic/PhysicsResultsB2G 